\title{
Evidence of Plasma Resonance Instability from Observations of Solar Decimetric Fine Structures
}

\author{
F.C.R. Fernandes ${ }^{1}$, H.S. Sawant ${ }^{1}$, J.R. Cecatto ${ }^{1}$, V. Krishan ${ }^{1,2}$, M. Karlický ${ }^{3}$, and R.R. Rosa ${ }^{1}$ \\ 1 - Instituto Nacional de Pesquisas Espaciais, C.P. 515, 12201-970, São José dos Campos, Brazil \\ 2 - Indian Institute of Astrophysics, Bangalore, 560 034, India \\ 3 - Ondřejov Observatory, Ondřejov, 251 65, Czech Republic
}

Received on 2 May, 2002. Revised version received on 13 August, 2002.

\begin{abstract}
The solar radio emissions in the decimetric frequency range (above $1000 \mathrm{MHz}$ ) are very rich in temporal and/or spectral fine structures. The Brazilian Solar Spectroscope (BSS) at INPE started operating in 1998. Since then, various types of fine structures, in the frequency range of (1000-2500) $\mathrm{MHz}$, with high time and frequency resolutions have been recorded. The observed bursts include dot-like emissions and chains of dots, lace bursts exhibiting intensity variations as a function of frequency and zebra pattern emissions. It is suggested that they can be generated by a plasma resonance instability mechanism. This model can explain many features of the observed fine structures.
\end{abstract}

\section{Introduction}

Initially, the solar radio bursts recorded at frequencies above $1000 \mathrm{MHz}$ were seen as featureless bursts believed to be generated by gyro-synchrotron emission mechanism (Kundu, 1965). However, with the constant improvement of the sensitivity and the temporal and the spectral resolutions of the solar radio spectrographs, many details of fine structures were revealed in decimetric radio emissions that are now being interpreted to be due to plasma emission mechanisms. The Brazilian Solar Spectroscope (BSS), at INPE (Sawant et al., 2000; 2001; Fernandes et al., 2000), belongs to this new generation of solar spectrographs.

Here, we present the fine structures classified as dot-like emissions (Sawant et al., 2002a), lace bursts (Jiřička et al., 2001; Karlický et al., 2001) and zebra pattern bursts (Sawant et al., 2002b) observed by BSS with high resolutions (Fernandes et al., 2001; 2002). Due to the similarities in their features, the three types of fine structures have been interpreted in a model based on the double plasma resonance instability first proposed by Zheleznyakov \& Zlotnik (1975) for zebra and tadpole patterns.

In section II we briefly describe the BSS and its main characteristics. In section III the observations and the main properties of the zebra, lace and dot emissions are presented. In section IV the double plasma resonance model is presented. The implications of the model on the generation of each type of fine structures are discussed in section V.

\section{Instrumentation}

The BSS was put into regular operation at INPE for systematic solar observations in 1998 (Sawant et al., 2001).
The BSS operates in conjunction with a 9 meter diameter polar mounted parabolic antenna, over the frequency range $1000-2500 \mathrm{MHz}$, with high time (10-50 ms) and frequency ( $3 \mathrm{MHz}$ ) resolutions. The data are recorded in up to 100 digital channels (Sawant et al., 2000), with absolute timing accuracy of 3 milliseconds. Thus, the BSS system has the capability to detect fine structures with narrow bandwidth and short durations (Fernandes et al., 2001).

\section{Observations}

The main characteristics of the decimetric radio emissions (dot-like, zebra patterns and lace burst) recorded by the BSS are summarized in the following sections.

\section{III.1 Dot-like emissions}

Dot-like emissions have been reported at decametermeter frequencies (34.0-35.5 MHz) by Sawant et al. (1976) and reviewed by Bhonsle et al. (1979). Allaart et al. (1990) have reported observations of dot-like emissions in the frequency range of 4000-8000 MHz. For the first time the BSS have recorded dot-like emissions in the frequency range of 1000-2500 MHz (Sawant et al., 2002a). The BSS recorded 51 groups of dot-like emissions, between 1999 and 2000. Out of these, we selected 20 groups for which we are able to determine the main parameters of the individual dot-like and/or their chains. Details of these 20 groups of dots are given in Table 1, including associated solar activity. Fig. 1 shows examples of isolated dot-like emissions randomly distributed. Fig. 2 shows examples of chains of dots with both drift rate orientations. Inverted-U and $U$ patterns of dots have been also recorded (Fig. 3 and Fig. 4). 
Table 1. Dot-like emissions recorded by BSS (updated from Sawant et al., 2002a).

\begin{tabular}{ccccccccc}
\hline Date & \multicolumn{2}{c}{ BSS Time (UT) } & \multicolumn{1}{c}{ Dot } & \multicolumn{2}{c}{ H-alpha Time (UT) } & Flare & Radio \\
yyyymmdd & Begin & End & Type & Begin & Max & End & Class & Assoc. \\
\hline 19990913 & $17: 00: 20$ & $17: 00: 22$ & RS + U chain & 1630 & 1645 & 1704 & C2.6 & III \\
19991007 & $15: 53: 31$ & $15: 53: 31$ & N chain & - & - & - & - & - \\
19991018 & $16: 26: 22$ & $16: 26: 26$ & iso + IU chain & 1555 & 1557 & 1620 & SF & \\
19991019 & $17: 56: 28$ & $17: 58: 07$ & isolated & 1757 & 1758 & 1820 & SF & - \\
19991020 & $16: 19: 42$ & $16: 19: 49$ & isolated & - & - & - & - & - \\
19991021 & $11: 53: 38$ & $11: 53: 38$ & V chain & - & - & - & - & - \\
19991028 & $18: 04: 26$ & $18: 04: 26$ & V chain & - & - & - & - & - \\
19991127 & $12: 08: 22$ & $12: 09: 49$ & isolated & - & - & - & X1.4 & - \\
20000417 & $14: 46: 46$ & $14: 46: 38$ & iso + chain & 1411 & - & 1709 & & CTM/1 \\
20000418 & $15: 00: 08$ & $15: 00: 64$ & isolated & - & - & - & - & - \\
20000508 & $15: 35: 19$ & $15: 35: 36$ & chain & A0530 & - & 1705 & & \\
20000606 & $15: 06: 21$ & $15: 06: 23$ & isolated & 1506 & 1519 & 1607 & & IV/3 \\
20000619 & $14: 55: 54$ & $14: 55: 55$ & iso & - & - & - & - & - \\
20000704 & $14: 58: 57$ & $14: 59: 01$ & isolated & 1458 & 1500 & 1516 & SF & \\
20000710 & $16: 21: 26$ & $16: 21: 33$ & isolated & 1355 & 1427 & 1623 & $1 N$ & \\
20000922 & $15: 17: 17$ & $15: 17: 38$ & iso + U chain & 0153 & 0513 & 2138 & & \\
20000923 & $14: 57: 08$ & $14: 57: 11$ & isolated & 0605 & - & 1624 & & CTM/1 \\
20000925 & $15: 18: 23$ & $15: 18: 43$ & isolated & 0520 & - & 1620 & & CTM/2 \\
20000929 & $16: 23: 33$ & $16: 23: 57$ & iso + U chain & & - & & & \\
20000929 & $16: 33: 46$ & $16: 33: 54$ & U chain & & - & & & \\
\hline
\end{tabular}

RS - Reverse slope drift; N - Normasl drift; V - Vertical; IU - Inverted U-type; U - U-type
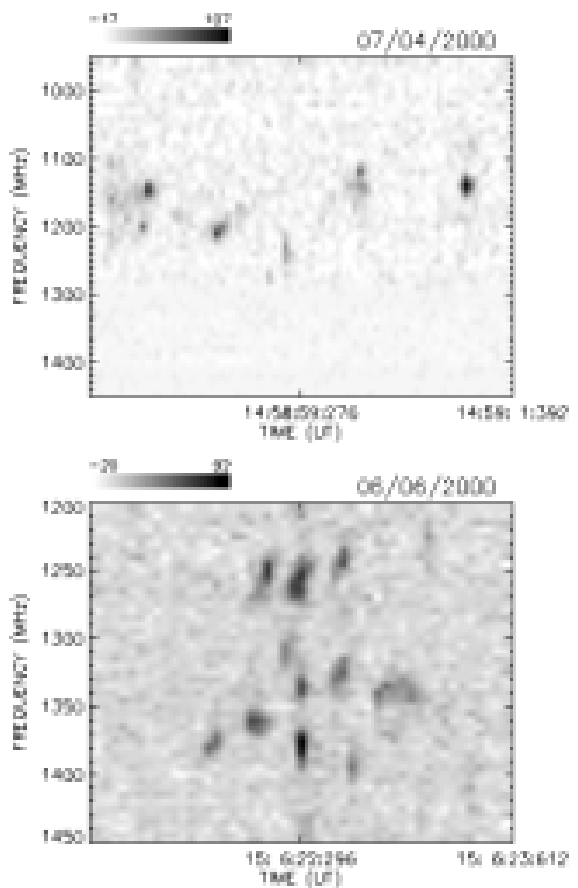

Figure 1. Dynamic spectra of groups of isolated dot-like emissions.
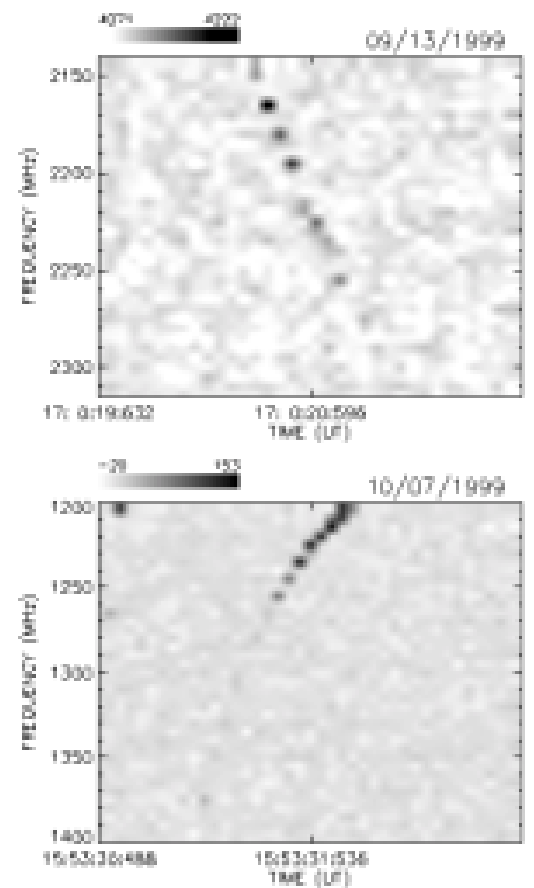

Figure 2. Dynamic spectra of chains of dots: (upper) with reverse slope frequency drift rate and (botom) with normal frequency drift rate. 


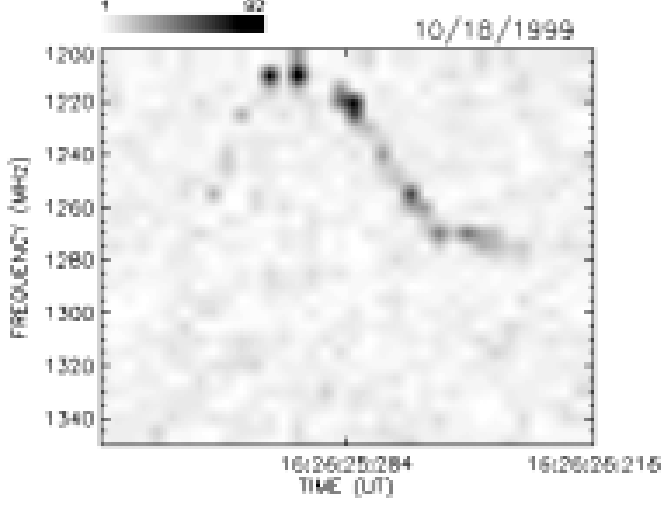

Figure 3. Dynamic spectrum of an inverted-U pattern chain of dots.

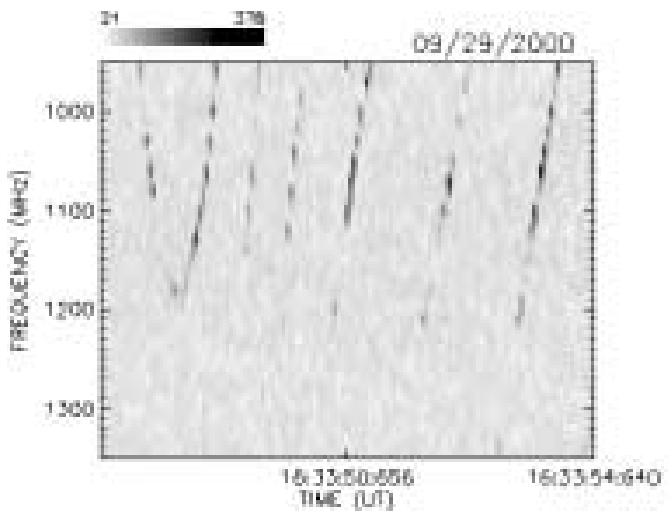

Figure 4. Dynamic spectrum of an U pattern chain of dots.

Mostly dot-like groups were associated with the rising portion of the impulsive phase of the associated flares, and many of them occur singly with a time separation of about 30 seconds. The number of individual dots in a group varies from 2 to about 30.

\section{III.2 Zebra patterns}

Metric zebra patterns have been observed in radio emissions for many decades (Slottje, 1972; Aurass and Chernov, 1983). More recently, Isliker and Benz (1994) reported zebra patterns in the decimetric range (1000-4000 MHz). Here, we report 8 zebra patterns observed by BSS in the frequency range of 1000-1700 MHz during the June 6, 2000 (15:00-17:00 UT) flare classified as X2.3. The list and parameters of the zebra patterns are shown in Table 2. Examples of the zebra patterns are shown in Fig. 5.
Table 2. Zebra patterns observed by BSS in June 06, 2000.

\begin{tabular}{|c|c|c|c|}
\hline \multicolumn{2}{|c|}{ BSS Time } & Frequency & No. of \\
\hline $\begin{array}{l}\text { Start } \\
\text { (UT) }\end{array}$ & $\begin{array}{l}\text { End } \\
\text { (UT) }\end{array}$ & $\begin{array}{l}\text { Range } \\
(\mathrm{MHz})\end{array}$ & $\begin{array}{l}\text { zebra } \\
\text { lines }\end{array}$ \\
\hline $15: 37: 41$ & $15: 37: 49$ & $1590-1685$ & 3 \\
\hline $15: 42: 53$ & $15: 42: 54$ & $1220-1265$ & 3 \\
\hline $15: 51: 32$ & $15: 51: 33$ & $1245-1385$ & 5 \\
\hline $15: 54: 42$ & $15: 54: 45$ & $1250-1395$ & 6 \\
\hline $16: 31: 20$ & $16: 31: 25$ & $1500-1690$ & 7 \\
\hline $16: 32: 13$ & $16: 32: 15$ & $1605-1685$ & 4 \\
\hline $16: 33: 07$ & $16: 33: 28$ & $1420-1500$ & 4 \\
\hline $16: 33: 53$ & $16: 33: 58$ & $1535-1680$ & 7 \\
\hline
\end{tabular}
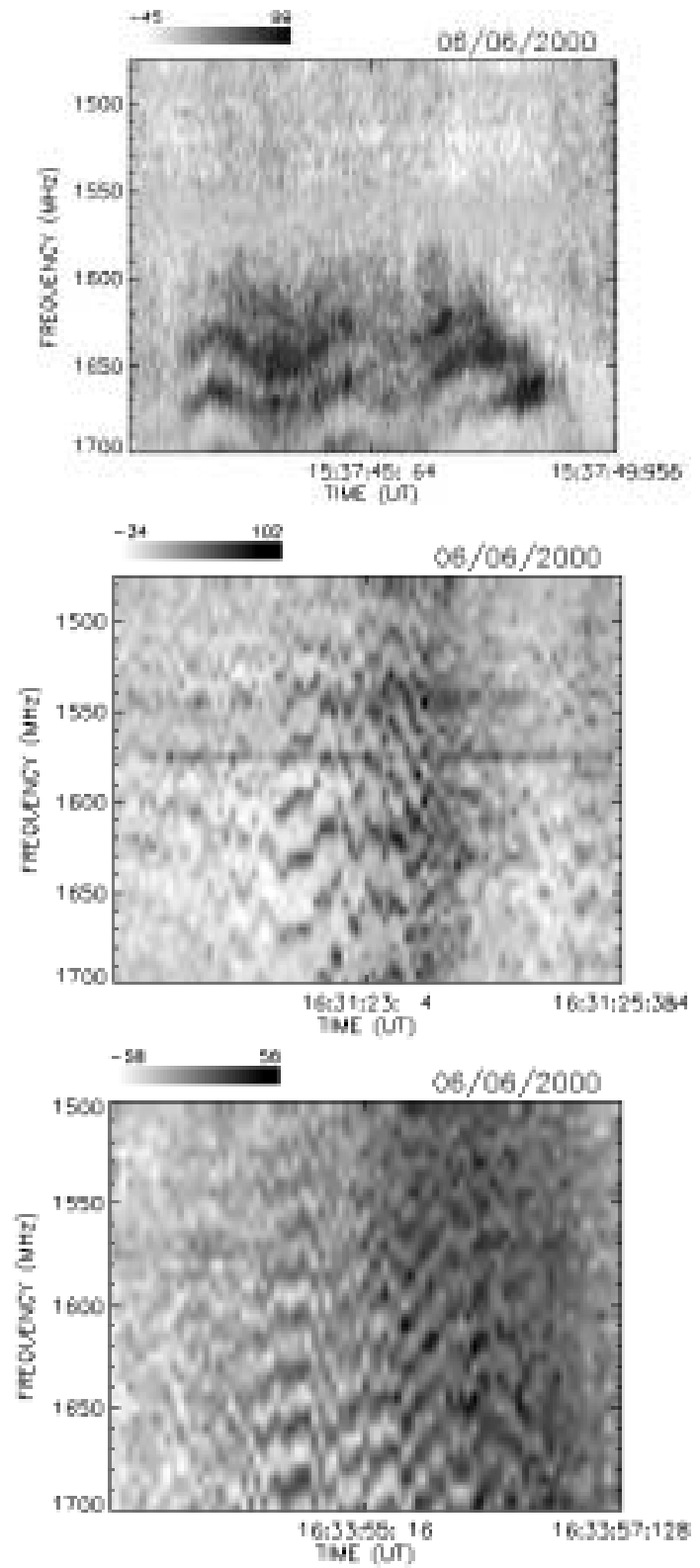

Figure 5. Dynamic spectra of radio emissions showing zebra patterns fine structures. 


\section{III.3 Lace bursts}

Radio bursts of long duration (minutes to hours) showing fast fluctuations of intensity with the frequency superimposed on the slow oscillations with typical regularity of the order of minutes have been recently designated as lace bursts by Jiřička et al. (2001). The lace type emissions are associated with CMEs or intense flares and type II bursts.

The BSS and the Ondřejov Spectrographs simultaneously recorded the lace bursts on August 17, 1999 during (14:47-17:04) UT (Karlický et al., 2001) (Fig. 6). The bursts have narrow band widths of about $50 \mathrm{MHz}$, large variations in intensity and frequency on the time scales of seconds and they are superimposed on a continuum, especially at low frequencies.

\section{Double plasma resonance instabil- ity model}

The phenomenological aspects and similarities of the observed radio fine structures (dot-like emissions, zebra patterns and lace bursts) can be explained assuming that they are generated at the positions in solar atmosphere where the following resonance condition is fulfilled:

$$
\omega_{U H}=\left(\omega_{p e}^{2}+\omega_{B e}^{2}\right)^{1 / 2}=s \omega_{B e},
$$

where $\omega_{U H}, \omega_{p e}$, and $\omega_{B e}$ are the upper hybrid, the electron plasma and the cyclotron frequencies, and $s$ is the integer harmonic number.

The electron Bernstein waves waves are generated by an anisotropic beam $\left(T_{\perp}>T_{\|}\right)$, where $T_{\perp}$ and $T_{\|}$are the temperatures of energetic electrons across and along the magnetic field, respectively. The beam anisotropy can be naturally formed due to an escape of fast electrons along the magnetic field.

Considering the resonance at the normal Doppler effect, i.e. $\omega-k_{\|} v_{\|}-s \omega_{B e}=0$, the growth rate, $\gamma$, of the unstable longitudinal waves is given by (Mikhailovsky, 1975)
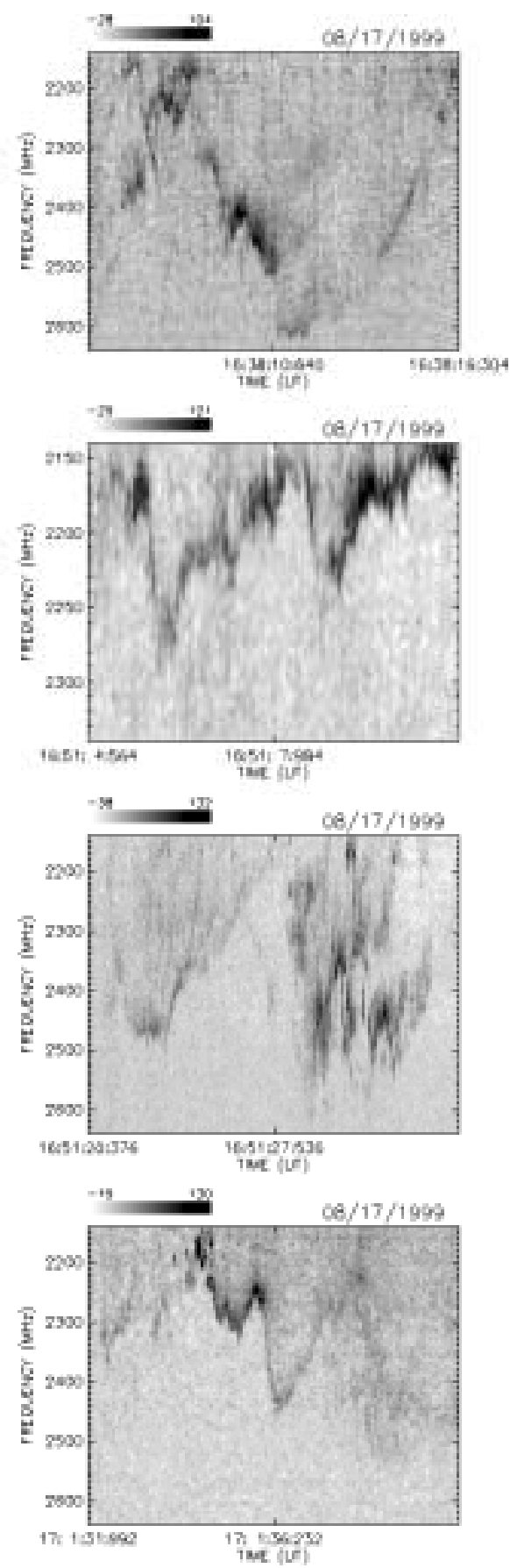

Figure 6. Dynamic spectra of different portions of a long duration emission showing lace bursts.

$$
\gamma=\frac{\sqrt{\pi} \alpha \omega^{3}}{v_{T e \|}^{3} k^{2}\left|k_{\|}\right|} \sum_{s=-\infty}^{\infty} I_{s} \exp \left(-z_{\perp}\right) \exp \left[-\left(\frac{\omega-k_{\|} v_{\|}-s \omega_{B e}}{k_{\|} v_{T e \|}}\right)^{2}\right] \times\left[\omega-k_{\|} v_{\|}-s \omega_{B e}\left(1-\frac{T_{\|}}{T_{\perp}}\right)\right]
$$


where $\alpha$ is the ratio between the beam density $n_{b}$ and the density of the background plasma $n, v_{T e \|}$ is the thermal electron velocity along the magnetic field, $k$ is the wave number, $I_{s}\left(k_{\perp} v_{\perp} / \omega_{B e}\right)$ is the modified Bessel function, $z_{\perp}=k_{\perp}^{2} T_{\perp} / m_{e} \omega_{B e}^{2}, m_{e}$ is the electron mass, $k_{\perp}$ and $k_{\|}$ are, respectively, the transverse and parallel components of the wave number.

It can be noted that the growth rate is inversely proportional to $k_{\|}$. In this case, the most effectively excited longitudinal waves are for $k_{\|} \ll k_{\perp}$. Thus, the frequency of these waves can be expressed as $\omega \approx\left(\omega_{p e}^{2}+\omega_{B e}^{2}\right)^{1 / 2}$ and the resonance condition become $\omega \approx s \omega_{B e}$ (Zheleznyakov \& Zlotnik, 1975; Ledenev et al., 2001).

In particular, it has been shown that the growth rate increases considerably when the double plasma resonance, i.e. when the Bernstein modes have frequencies equal to the frequency of the upper hybrid mode. In the inhomogeneous environment the double resonance takes place only at certain positions where the electron density and magnetic field have the right values to satisfy the resonance condition. This is the reason for the discrete dot emission. Using this model, we can determine the physical parameters of the ambient coronal plasma. From the condition that the growth rate exceeds the damping of the electromagnetic waves due to collisional and cyclotron absorption, we can determine the parameters of the exciting agency such as the anisotropic electron beam. We find that electrons with perpendicular velocity of $\simeq 0.2 \mathrm{c}$ can excite the Bernstein modes with growth rate much larger than the damping rate.

The generated upper hybrid waves are then transformed into observable electromagnetic waves of frequency $\omega_{e l}$ (radio emission) at the fundamental or the second harmonic frequency, i.e. $\omega_{e l} \approx \omega_{U H}$ and/or $\omega_{e l} \approx 2 \omega_{U H}$, respectively.

Using the resonance condition for $s$-harmonic component (zebra line or dot) one can write

$$
\omega_{s}=\frac{\omega_{p e s}}{\left(1-\frac{1}{s^{2}}\right)^{1 / 2}},
$$

where $\omega_{p e s}$ is the electron plasma frequency at $s$-resonance. Thus, the ratio of neighbouring components frequencies is given by

$$
\frac{\omega_{s}}{\omega_{s+1}}=\frac{\omega_{p e s}}{\omega_{p e s+1}}\left[\frac{s^{3}(s+2)}{(s+1)^{3}(s-1)}\right]^{1 / 2} .
$$

Assuming that the density decreases with height much slower than the magnetic field, we take roughly $\omega_{\text {pes }} \approx$ $\omega_{\text {pes }+1}$. Thus, the ratios between neighbouring components will be $\omega_{2} / \omega_{3} \approx 1.09 ; \omega_{3} / \omega_{4} \approx 1.03, \omega_{4} / \omega_{5} \approx 1.01$, and so on. These values are minimal and they are in good agreement with those obtained by zebra patterns components.

\section{Summary and discussions}

The mechanism based on double plasma resonance instability, first suggested by Zheleznyakov \& Zlotnik (1975) is pre- sented and used to explain the generation of fine structures, such as dot-like emissions, zebra patterns and lace bursts recorded at decimetric frequencies by the Brazilian Solar Spectroscope with high time and frequency resolutions.

Dot-like emissions: The model can explain not only individual dots, but also the chains of dots. For individual dots, the anisotropic beam instability generates the plasma waves only in one resonance position. However, for the chains, the beam along its trajectory generates dots in several resonance positions (several $s$-harmonics). The higher $s$ corresponds to the higher height of the dot source in the solar atmosphere. Also, the model can explain the regular decrease of the ratio of the neighbouring harmonics.

Zebra patterns: The model explains not only the zebra pattern generation, but also the small ratios of zebra pattern frequencies, their variability and their tendency to decrease towards lower frequency. It means the generated upper-hybrid waves are transformed to the electromagnetic waves of the same frequency as well as its second harmonic.

Lace bursts: The characteristic rapid frequency variations of the lace bursts line can result due to generation in a plasma with rapidly changing plasma parameters (density or/and temperature). The accompanying continua can be a superposition of many weak lace lines generated by some additional instabillities in the disturbed flare plasma.

\section{Acknowledgements}

Thanks are due to FAPESP for supporting the visit of M. Karlický (01/00144-5) and V. Krishan (01/06031-8) to INPE. F.C.R. Fernandes thanks FAPESP for receiving a scholarship (99/10529-1). The authors would like to thank the unknown referee for the critical comments and suggestions which helped to improve the quality of the paper.

\section{References}

Allaart, M.A.F.; Nieuwkoop, J.; Slottje, C.; Sondaar, L.H., Solar Phys. 130, 183 (1990).

Aurass, H.; Chernov, G.P., Solar Phys. 84, 339 (1983).

Bohnsle, R.V.; Sawant, H.S.; Degaonkar, S.S., Space Science Rev. 24, 259 (1979).

Fernandes, F.C.R.; Cecatto, J.R.; Neri, J.A.C.F.; Faria, C.; Martinon, A.R.F.; Rosa, R.R.; Mesquita, F.P.V.; Portezani, V.A.; Andrade, M.C.; Alonso, E.M.B.; Vats, H.O.; Sawant, H.S., Boletim da SAB, 20, 33 (2000).

Fernandes, F.C.R.; Cecatto, J.R.; Neri, J.A.C.F.; Silva, M.J.B.; Rosa, R.R.; Faria, C.; Andrade, M.C.; Alonso, E.M.B.; Martinon, A.R.F.; Dobrowolski, K.M.; Barbosa, T.M.; Freitas, D.C.; Nascimento, V.F.; Reis, A.L.; Sawant, H.S., Boletim da SAB, 21, 49 (2001).

Fernandes, F.C.R.; Sawant, H.S.; Cecatto, J.R.; Neri, J.A.C.F.; Martinon, A.R.F.; Freitas, D.C., Ap. J. Supp. Ser. 2002. Submitted.

Isliker, H.; Benz, A.O., Astron. Astrophys. Suppl. Series, 104, 145 (1994).

Jiřička, K.; Karlický, M.; Mészarosova, H.; Snízek, V., Astron. Astrophys. 375, 243 (2001). 
Karlický, M.; Barta, M.; Jiřička, K.; Mészarosova, H.; Sawant, H.S.; Fernandes, F.C.R.; Cecatto, J.R., Astron. Astrophys. 375, 683 (2001).

Kundu, M.R., Solar Radio Astronomy, Interscience Publ., 1965.

Ledenev, V.G.; Karlický, M.; Yan, Y.; Fu, Q., Solar Phys. 202, 71 (2001).

Mikhailovsky, A.B., Theory of Plasma Instabilities Vol. I, Nauka, Moscow, 1975.

Sawant, H.S.; Bhonsle, R.V.; Alurkar, S.K., Solar Phys. 50, 481 (1976).

Sawant, H.S.; Subramanian, K.R.; Faria, C.; Stephany, S.; Fernandes, F.C.R.; Cecatto, J.R.; Rosa, R.R.; Portezani, V.A.; Mesquita, F.P.V.; Alonso, E.M.B., Astronomical Society of the Pacific Conference Series, 206, 347 (2000).
Sawant, H.S.; Subramanian, K.R.; Faria, C.; Fernandes, F.C.R.; Sobral, J.H.A.; Cecatto, J.R.; Rosa, R.R.; Vats, H.O.; Neri, J.A.C.F.; Alonso, E.M.B.; Mesquita, F.P.V.; Portezani, V.A.; Martinon, A.R.F., Solar Phys. 200, 167 (2001).

Sawant, H.S.; Fernandes, F.C.R.; Cecatto, J.R.; Vats, H.O.; Neri, J.A.C.F.; Portezani, V.A.; Martinon, A.R.F.; Karlický, M.; Jiřička, K.; Mészarosova, H., Adv. Space Res. 29, 349 (2002a).

Sawant, H.S.; Karlický, M.; Fernandes, F.C.R.; Cecatto, J.R., Proc. Yohkoh 10th Anniversary Meeting, COSPAR Colloquia Series 13 (2002b).

Slottje, C., Proc. 2nd CESRA Meeting, 88, 1972.

Zheleznakov, V.V.; Zlotnik, E.Ya., Solar Phys. 44, 461 (1975). 